\title{
Retracted: Microwave Mediated Organic Reaction: A Convenient Approach for Rapid and Efficient Synthesis of Biologically Active Substituted 1,3-Dihydro-2H-indol-2-one Derivatives
}

\author{
Journal of Nanomaterials \\ Received 16 December 2015; Accepted 16 December 2015 \\ Copyright (C) 2015 Journal of Nanomaterials. This is an open access article distributed under the Creative Commons Attribution \\ License, which permits unrestricted use, distribution, and reproduction in any medium, provided the original work is properly \\ cited.
}

The paper titled "Microwave Mediated Organic Reaction: A Convenient Approach for Rapid and Efficient Synthesis of Biologically Active Substituted 1,3-Dihydro-2H-indol-2-one Derivatives" [1], published in Journal of Nanomaterials, has been retracted as it was accepted for publication on the basis of peer review reports that were submitted from fraudulent reviewer accounts.

In late 2014, a number of publishers discovered widespread abuse of the peer review process, including cases of identity theft and faked review reports. In July 2015, Hindawi concluded an extensive investigation into peer review fraud and identified a number of articles that had been accepted on the basis of fraudulent peer review reports. In accordance with the recommendations of the Committee on Publication Ethics (COPE), Hindawi sent these manuscripts for re-review using independent Editorial Board Members. Following this re-review process, this article has been retracted as it was deemed unsuitable for publication.

\section{References}

[1] J. Panda, B. M. Sahoo, N. K. Mishra, S. K. Padhi, and J. Mishra, "Microwave mediated organic reaction: a convenient approach for rapid and efficient synthesis of biologically active substituted 1,3-dihydro-2H-indol-2-one derivatives," Journal of Nanomaterials, vol. 2013, Article ID 272598, 8 pages, 2013. 